\title{
EchoGéo
}

36 | 2016

Stratégies de villes et "modèles urbains"

\section{Circulation d'un modèle urbain "alternatif" ?}

Le cas de l'urbanisme tactique et de sa réception à Paris

\section{Nicolas Douay and Maryvonne Prévot}

\section{(2) OpenEdition}

\section{Journals}

Electronic version

URL: https://journals.openedition.org/echogeo/14617

DOI: $10.4000 /$ echogeo. 14617

ISSN: 1963-1197

\section{Publisher}

Pôle de recherche pour l'organisation et la diffusion de l'information géographique (CNRS UMR 8586)

\section{Electronic reference}

Nicolas Douay and Maryvonne Prévot, "Circulation d'un modèle urbain "alternatif" ?", EchoGéo [Online], 36 | 2016, Online since 30 June 2016, connection on 10 August 2021. URL: http://

journals.openedition.org/echogeo/14617 ; DOl: https://doi.org/10.4000/echogeo.14617

This text was automatically generated on 10 August 2021.

EchoGéo est mis à disposition selon les termes de la licence Creative Commons Attribution - Pas d'Utilisation Commerciale - Pas de Modification 4.0 International (CC BY-NC-ND) 


\section{Circulation d'un modèle urbain "alternatif" ?}

Le cas de l'urbanisme tactique et de sa réception à Paris

Nicolas Douay and Maryvonne Prévot

1 Dans un contexte de globalisation, l'évolution des politiques d'aménagement se caractérise depuis plusieurs décennies par des dynamiques de standardisation et d'homogénéisation (Harvey, 1989; Hall et Hubbard, 1996; Moulaert et al., 2005) qui mettent en avant des «bonnes pratiques» (Espaces et Sociétés, 2007) à diffuser. Ces pratiques de référence renvoient à des caractéristiques substantielles des politiques urbaines dans leur contenu commun, mais aussi à des caractéristiques procédurales censées définir la «bonne gouvernance ». Ainsi, des modèles urbains classiques, voire dominants apparaissent et tentent de répondre aux dogmes du néolibéralisme (Brenner et Theodore, 2002; Béal et Rousseau, 2008). Ces solutions standardisées mêlent bien souvent un processus de renouvellement urbain par le développement de nouveaux quartiers d'affaires ou d'incubateurs numériques, sur fond de stratégie de marketing urbain associant tourisme, culture et sport, le plus souvent dans le cadre de l'organisation de grands évènements. L'exemple de Bilbao en Espagne est caractéristique de ce phénomène (Masboungi, 2001 ; Gravari-Barbas \& Jacquot, 2007; Douay et Duaso, 2008).

2 Ces pratiques urbaines mainstream ne sont pas reproduites en totalité mais servent plutôt de référence dans des situations d'adaptation d'un espace à un autre (Peyroux, 2014). Les notions de «Policy Transfer» (Dolowitz et Marsh 1996; Dolowitz et Marsh, 2000) ou de mobilité des politiques urbaines «Urban policies mobility » renvoient ainsi à un processus par lequel les savoirs sur les arrangements administratifs et institutionnels utilisés dans un système politique donné (passé ou présent) sont utilisées pour développer des politiques et des arrangements dans un autre contexte. Les échanges s'appuient sur différentes communautés d'esprit, de pratiques ou d'expertises (Peck et Theodore 2010 ; Stone, 2004). Dans le contexte de métropolisation, ces transferts de modèles se focalisent essentiellement sur les villes. Elles sont les 
nœuds stratégiques de connaissances et d'échanges, avant les États (McCann, 2011; McCann et Ward, 2011; McCann et Ward, 2012).

Dans cet article, nous proposons d'étudier un aspect encore très peu développé dans la littérature (Capelli, 2013; Crombez, 2014), à savoir le tactical urbanism. À l'image du Québec nous avons choisi de traduire l'expression en français par «urbanisme tactique ». Au-delà d'une transformation de l'espace plus ou moins temporaire, le tactical urbanism renvoie avant tout à un engagement citoyen pour lequel l'aménagement est une pratique collective qui mobilise souvent les ressorts de l'art et de l'évènementiel. Cette pratique sociale de l'urbanisme est envisagée ici comme un modèle urbain alternatif. En 2003, la revue Métropoles (2013) ${ }^{1}$ définissait ce caractère " alternatif» comme «l'ensemble des initiatives, des démarches ou des projets soutenus par des municipalités et qui cherchent à organiser un développement urbain s'éloignant des canons de l'entrepreneurialisme» en se plaçant dans une perspective "bottom-up ». Si traditionnellement, les politiques urbaines sont plutôt descendantes et "nonmarchandes » car elles ne font pas des mécanismes marchands leur principal vecteur d'organisation. (cf. points 1 et 2 de l'appel), ce mouvement de l'urbanisme tactique vient bien proposer des modalités alternatives au développement urbain. Du point de vue procédural, il propose de transformer et dynamiser l'espace public urbain grâce à des initiatives citoyennes. En ce sens il participe à la reconnaissance du pluralisme des acteurs et au développement des démarches participatives dans l'aménagement. Du point de vue substantiel, il s'inscrit dans une recherche de valeurs alternatives à celles de compétitivité et d'attractivité économiques comme référentiel dominant des politiques urbaines actuelles. Il propose des interventions légères et fréquemment réversibles, éloignées des modes d'intervention lourds des pratiques traditionnelles de l'urbanisme qui favorisent en particulier la construction d'infrastructures au profit des automobiles.

4 La méthodologie de cette contribution s'appuie sur une observation des pratiques militantes en ligne et hors-ligne depuis six années de façon assez continue. Une observation sur le terrain de ces transformations urbaines a d'abord été réalisée à Paris, Lille, Bruxelles et Montréal avec la réalisation d'une dizaine d'entretiens semidirectifs menés tant avec les militants acteurs de ces transformations qu'avec les usagers spectateurs de ces aménagements. La réalisation d'une revue de presse sur ces différentes villes ainsi que l'encadrement de travaux d'étudiants plongés dans des démarches d'observation participante au cours de leurs stages professionnels de fin d'études de master d'aménagement et d'urbanisme dans diverses structures, en France, en Belgique ou au Canada, ont permis de compléter la collecte des données (Bigand, 2010 ; Crombez, 2014 ; Denais, 2012 ; Duval, 2014 ; Raynaud-Desmet, 2011). Enfin pour les besoins de cet article, une étude de l'espace numérique a été menée de façon systématique via l'analyse des manuels d'urbanisme tactique qui s'y trouvent et servent de base à la définition et circulation du modèle. Les réseaux sociaux associés à ces pratiques militantes ont aussi été une source importante d'information, notamment les comptes Twitter et Facebook des principaux groupes et leaders pour lesquels nous avons mobilisé des outils d'analyse de ces big data (observation du nombre d'abonnés, de messages, popularité et contenu des messages). Ces ressources numériques ont donc fait l'objet d'une analyse textuelle afin d'identifier la circulation des concepts ainsi que les agents de ces circulations. 
5 L'apparition et la circulation internationale de la notion de tactical urbanism feront l'objet d'une première partie puis l'étude du cas parisien permettra de pointer les modalités de réception et d'institutionnalisation de ce mouvement dans la capitale française; enfin, le passage d'un modèle alternatif à un nouveau modèle dominant sera questionné.

\section{Apparition et circulation du tactical urbanism}

6 Le tactical urbanism est un modèle urbain alternatif qui affirme se situer, en partie au moins, dans la lignée historique des mouvements sociaux urbains et va se diffuser principalement grâce aux outils de communication numérique qui vont créer un réseau de pratiques militantes à l'échelle mondiale.

\section{Un urbanisme citoyen}

7 L'émergence de cet urbanisme tactique s'inscrit donc dans l'héritage des mouvements sociaux urbains des années 1960 aux années 1980. Certains de ses adeptes disent puiser leur inspiration dans les écrits théoriques d'Henri Lefebvre $(1968,1974)$ et des situationnistes (Simay, 2008 ; Douay et Prévot, 2014) ou encore dans ceux de Jane Jacobs (1961), Kevin Lynch, William Whythe ${ }^{2}$ (1980) ou Michel de Certeau (1990). Ce dernier mobilise tout particulièrement cette notion de tactique: "Les tactiques sont des procédures qui valent par la pertinence qu'elles donnent au temps - aux circonstances que l'instant précis d'une intervention transforme en situation favorable, à la rapidité de mouvements qui changent l'organisation de l'espace, aux relations entre moments successifs d'un "coup", aux croisements possibles de durées et de rythmes hétérogènes " (1990, p 62).

8 Les praticiens locaux explicitent ces différentes filiations: "L'art fait partie de l'espace public comme d'autres fonctions et usages, l'art vient apporter un plus, il dépasse l'espace public comme un lieu fonctionnaliste pour révéler d'autres choses. Ça aussi dépend de ce qu'on entend par art? Art public? Performance? Le designer urbain s'intéresse à faire des lieux qui peuvent accueillir ces appropriations artistiques, laisser les choses émerger de façon spontanée. Donner le droit d'évoluer dans le temps. (...) Dans le Park(ing) Day, l'idée est moins de se révéler comme un artiste que comme un citoyen engagé avec une identité de revendication autour d'une expérience sociale. C'est de l'art et de la politique en même temps. On descend des situationnistes, lorsqu'on sortait de l'art contemporain rigide» (Entretien avec une des fondatrices de l'Association de Design Urbain du Québec (ADUQ) le 2 août 2013 - cité dans Douay et Prévot, 2014, p 19).

9 Tous à leur manière opposent planification urbaine stratégique et urbanisme tactique, notamment dans leur rapport à la temporalité de l'action. Tous se sont emparés des concepts d'aliénation, de critique de la vie quotidienne mais aussi de créativité, de détournement et d'expérimentation comme moyens par lesquels la démocratie pourrait se frayer un chemin et une place dans les fissures ou les interstices de la planification, qu'elle soit matérielle, financière, technocratique ou économique. Tous veulent remettre le regard humain et les habitants au centre des débats et de l'action de reconquête d'une centralité et d'une vie sociale urbaine harmonieuse.

10 En Amérique du Nord, les militants revendiquent aussi un positionnement dans la lignée des travaux de Jane Jacobs et des mouvements sociaux urbains des années 1960 
et 1970. Différentes organisations vont promouvoir une vision alternative des politiques urbaines (Capelli, 2013). L'organisation «Project for Public Spaces » (PPS) voit ainsi le jour en 1975, fondé par Fred Kent, un élève de William « Holly » Whyte. Géographe de formation, il s'engage rapidement au profit de différentes causes communautaires à New York et il se consacre maintenant à temps-plein au développement de PPS. Ce projet a pour objectif d'aider «les gens à créer et à maintenir des espaces publics qui renforcent les communautés (...) d'aider les citoyens à transformer leurs espaces publics en lieux vitaux qui mettent en valeur les atouts locaux, stimulent le rajeunissement et servent les besoins communs $»^{3}$ (traduit à partir du site web). En 2015, PPS indique avoir réalisé des projets auprès de 3000 communautés dans 43 pays. Elle rassemble plus de 900 membres et fait office de référence pour l'ensemble de la communauté des citoyens engagés dans la transformation des espaces urbains. Cette approche est souvent résumée comme étant du "Placemaking " (Jolé et Tonnelat, 2010). L'expression de Placemaking n'est pas facile à traduire en français mais nous pourrions avancer les termes de «fabrique des espaces publics ». Il s'agit donc d'une appropriation citoyenne des espaces publics par la communauté depuis leur conception jusqu'à leur gestion.

11 Depuis le tournant $\mathrm{du} \mathrm{XXI}^{\mathrm{e}}$ siècle, ces initiatives se multiplient et l'expérience circule d'un espace à un autre. Elles portent différents noms : « Guerilla urbaine » (Hou, 2010), « urbanisme Do it your self» (DIY) (Finn, 2014 ; Douglas, 2014 ; Iveson, 2013 ; Sawhney et al., 2015.), « urbanisme généreux» (Merker, 2010), «activisme urbain » (Douay et Prévot, 2012), « acuponcture urbaine » (Lerner, 2007; Casagrande, 2010), " urbanisme pop-up ", " urban hacking " ou encore "urbanisme tactique " (Lydon, 2010; Lydon et Garcia, 2015). Ces pratiques, sous leurs divers intitulés, relèvent de l"urbanisme tactique, expression la plus répandue et que nous reprenons donc à notre compte. À partir des manuels (Lydon et Garcia, 2015) que cette communauté du tactical urbanism fait circuler via les réseaux sociaux, nous avons établi une liste (voir illustration 1) des actions possibles.

Illustration 1 - Typologie des interventions

\begin{tabular}{|l|l|}
\hline Expression d'origine & Traduction \\
\hline Open Streets & La piétonisation temporaire de rue \\
\hline Play Steets & L'animation de rue \\
\hline Build a better Block & Promouvoir un meilleur quartier \\
\hline Guerilla Gardening & Végétaliser \\
\hline Pop-up Retail & Commerce temporaire \\
\hline Pavement to Plazas & La reconquête de place \\
\hline Pavement to Parks & La création de parcs \\
\hline Pop-up Cafés & Café temporaire \\
\hline Depave & Retirer l'asphalte \\
\hline
\end{tabular}




\begin{tabular}{|l|l|}
\hline Chair Bombing & Installer du mobilier \\
\hline Food Carts/Trucks & Installer des camions servant de la nourriture \\
\hline Site Pre-Vitalization & Revitalisation temporaire d'un espace \\
\hline Pop-up Town Hall & Espace de discussion temporaire \\
\hline Informal Bike Parking & Espace de stationnement pour vélos \\
\hline Intersection Repair & Aménagement des intersections routières \\
\hline Ad-Busting & Retirer les espaces publicitaires \\
\hline Reclaimed Setbacks & Aménagement des trottoirs et des jardinets des maisons sur rue \\
\hline Park Mobile & Parcs et bancs temporaires \\
\hline Weed Bombing & Attirer l'attention sur l'entretien des espaces publics \\
\hline Mobile Vendors & Offrir des espaces commerciaux mobiles \\
\hline Micro-Mixing & Offrir des espaces commerciaux en colocation \\
\hline Park-Making & Aménager de nouveaux parcs \\
\hline Camps & Camp temporaire \\
\hline Park(ing) Day & Transformer les espaces de stationnement \\
\hline
\end{tabular}

Source : Lydon et Garcia, 2015.

12 Toutes ces initiatives ne sont pas forcément subversives mais, dans leurs contextes d'application, elles portent la revendication collective d'une pratique alternative de l'urbanisme. En ce sens elles constituent un mouvement social urbain qui se matérialise par la volonté d'une réappropriation de l'espace par le piéton et ce par l'entremise d'un matériel de base (chaises, tables, etc.) simplissime et des mises en scènes peu coûteuses. Toutes illustrent cette volonté de remettre l'humain au centre du fonctionnement de l'espace. "Improving the liveability of our towns and cities... " (Lydon, 2011): tel est l'objectif principal de l'urbanisme tactique qui se définit avant tout comme un projet citoyen qui remet donc l'espace public au cœur de la vie urbaine, en insistant sur la dimension ludique, artistique et temporaire des actions menées. Créer des projets aisément compréhensibles et appropriables par des communautés à travers le monde, telle est l'ambition affichée de faire ainsi œuvre d'éducation populaire, de pédagogie à la planification et l'environnement urbains, de manière interactive et festive, en faisant de ces actions des outils d'émancipation et d'empowerment (Bacqué \& Biewener, 2013), créant matière et souffle à une identité communautaire partagée.

13 Les interventions urbaines, le plus souvent ponctuelles, éphémères et artistiques proposées par ces diverses communautés connectées se réclamant du tactical urbanism correspondent aussi à cette "volonté d'agora » expliquée par Paul Ardenne (2002) dans ses travaux. Il y définit l'artivisme, à savoir un néologisme combinant les notions d'art et 
d'activisme, le plus souvent urbain, comme un art d'accompagnement des mutations urbaines et de démocratisation de l'art tout à la fois.

\section{Un réseau global et connecté}

14 La popularisation du terme d'urbanisme tactique doit beaucoup à Mike Lydon ${ }^{4}$, fondateur hyperconnecté du réseau regroupé sous le vocable de Street Plans Collaborative $^{5}$. C'est lui qui rédige, promeut et diffuse depuis 2010 le manuel «Tactical urbanism: Short Term action, Long Term Change» (Lydon et Garcia, 2015), dont la circulation des contenus s'effectue par l'intermédiaire des réseaux sociaux notamment la page Facebook ${ }^{6}$ où Mike Lydon a 1681 amis et plus encore la page Twitter ${ }^{7}$. Ce vecteur présente un mode d'interaction plus ouvert avec 14200 messages et 10800 abonnées (février 2016). C'est à ce jour la référence reconnue en termes de littérature professionnelle. Elle est diffusée librement sur le web et des traductions progressives en différentes langues ont vu le jour grâce aux contributions des membres de cette communauté. Le succès est tel, sur le territoire nord-américain d'abord, à l'échelle internationale ensuite, que l'urbanisme tactique était présent au sein du pavillon officiel des États-Unis à la Biennale de Venise en 2012, puis au Congrès du Nouvel Urbanisme $^{8}$ (New Urbanism) à Buffalo en 2014, ou encore au MOMA de New-York au travers d'une exposition intitulée "Uneven Growth: Tactical urbanism for expanding cities $»^{9}$ (Crombez, 2014). Il existe d'autres figures de diffusion du concept, proche, d'acupuncture urbaine ${ }^{10}$ telles que l'architecte et sociologue finlandais Marco Casagrande (3 895 amis sur Facebook) ${ }^{11}$ ou Jaime Lerner ${ }^{12}$ (7 391 amis sur Facebook et 3604 abonnés sur Twitter). Cet ancien maire de Curitiba, architecte-urbaniste est l'auteur d'un ouvrage éponyme dont la version numérique est disponible à bas coût.

$\mathrm{Au}$-delà du rôle des leaders qui sont les principaux agents de diffusion de ce modèle urbain alternatif, l'étude des mécanismes de diffusion de l'urbanisme tactique met en évidence une forte logique de réseau qui s'appuie sur les ressources numériques du Web 2.0, notamment les réseaux sociaux (voir illustration 2). L'informalité et l'horizontalité de ces réseaux sociaux sont fréquemment promues comme garantes d'une plus grande ouverture et accessibilité aux citoyens "lambda». Les travaux de Saskia Sassen $(2001,2011)$ viennent souligner l'influence des nouvelles technologies de l'information et de la communication qui, en abolissant les frontières et en créant des communautés, viennent connecter de nombreux individus à l'échelle de toute la planète : "Through the Internet, local initiatives become part of a global network of activism without losing the focus on specific local struggles » (Sassen, 2001, p. 416). L'espace local est alors une hybridation entre local et global, des actions locales peuvent prendre une signification globale.

Illustration 2 - Principaux comptes Twitter du mouvement de tactical urbanism

\begin{tabular}{|l|l|l|l|}
\hline Nom du compte & Adresse du compte & Nombre d'abonnés & Nombre de messages \\
\hline Proj 4 Public Spaces & @PPS_Placemaking & 56400 & 19300 \\
\hline Mike Lydon & @MikeLydon & 10800 & 14200 \\
\hline Street Plans & @StreetPlans & 10400 & 3876 \\
\hline
\end{tabular}




\begin{tabular}{|l|l|l|l|}
\hline Open Streets Project & @Open_Streets & 4865 & 2257 \\
\hline Center4ActiveDesign & @active_design & 1163 & 1534 \\
\hline Adaptive Cities & @AdaptiveCities & 10300 & 2240 \\
\hline CIRD & @rural_design & 1343 & 1334 \\
\hline PARK(ing) Day & @parkingday & 2653 & 247 \\
\hline Engaging Cities & @EngagingCities & 41300 & 4809 \\
\hline Pop-Up City & @popupcity & 17700 & 4727 \\
\hline Enabling City & @enablingcity & 17000 & 9576 \\
\hline
\end{tabular}

Source : Twitter, février 2016. connaissent donc une mise en réseau à l'échelle globale. Celles-ci seraient la preuve d'un renouveau de l'engagement civique et témoigneraient aussi d'un regain d'intérêt pour la vie publique: "The spirit of entrepreneurship combined with the aspiration of civic renewal $\aleph^{13}$ (Lydon, 2011). Couplée à la culture en expansion du Do It Yourself (Faites-le vous-même), l'apport de la technologie numérique a, semble-t-il, ainsi favorisé la popularisation des interventions d'urbanisme tactique.

tectical urbanism ferait donc passer les processus de revitalisation urbaine d'une opération exogène, imposée par les techniciens et les élus, à une opération endogène dans laquelle les habitants jouent un rôle primordial et rendraient la ville plus open source que jamais. Par le numérique et les réseaux sociaux, l'urbanisme tactique ouvrirait donc ainsi le système de production ou de fabrique de la ville aux citoyens ordinaires invitant ces derniers à y entrer pour contribuer à le transformer, à l'améliorer. Chiara Camponeschi utilise le concept de «Place-Bases Creative Solving Problem $»^{14}$ que l'on pourrait traduire par un processus créatif et localisé de résolution de problèmes, urbains en l'occurrence. De son côté, le géographe Olivier Mould (2014) use à dessein de la métaphore informatique - du virus cette fois - pour revisiter les écrits de Michel de Certeau. La tactique serait ainsi une forme de virus qui s'insinue, intègre l'environnement urbain - assimilé à un disque dur - pour le déconstruire et le transformer subrepticement et non pour le détruire.

\section{Le Parking Day modèle et label international de cet urbanisme tactique open source}

Le Park(ing) Day est certainement l'expérience la plus largement diffusée sur le globe, dans sa partie occidentale principalement. En 2005, REBAR ${ }^{15}$, un collectif d'artistes, designers, paysagistes et activistes installés à San Francisco décide de convertir un espace de stationnement en un espace vert, ludique, artistique et citoyen «en miniature ", le temps d'une journée. L'expérience, qui n'avait pas forcément vocation à être reproduite dans l'espace ou dans le temps, va très rapidement circuler sur le web 
avec pour support la photo d'une installation (illustration 3) et faire du «Park(ing) Day $»^{16}$ un évènement spécifique et clairement identifié à l'échelle internationale.

Illustration 3 - La première édition du Park(ing) Day à San Francisco, 2005

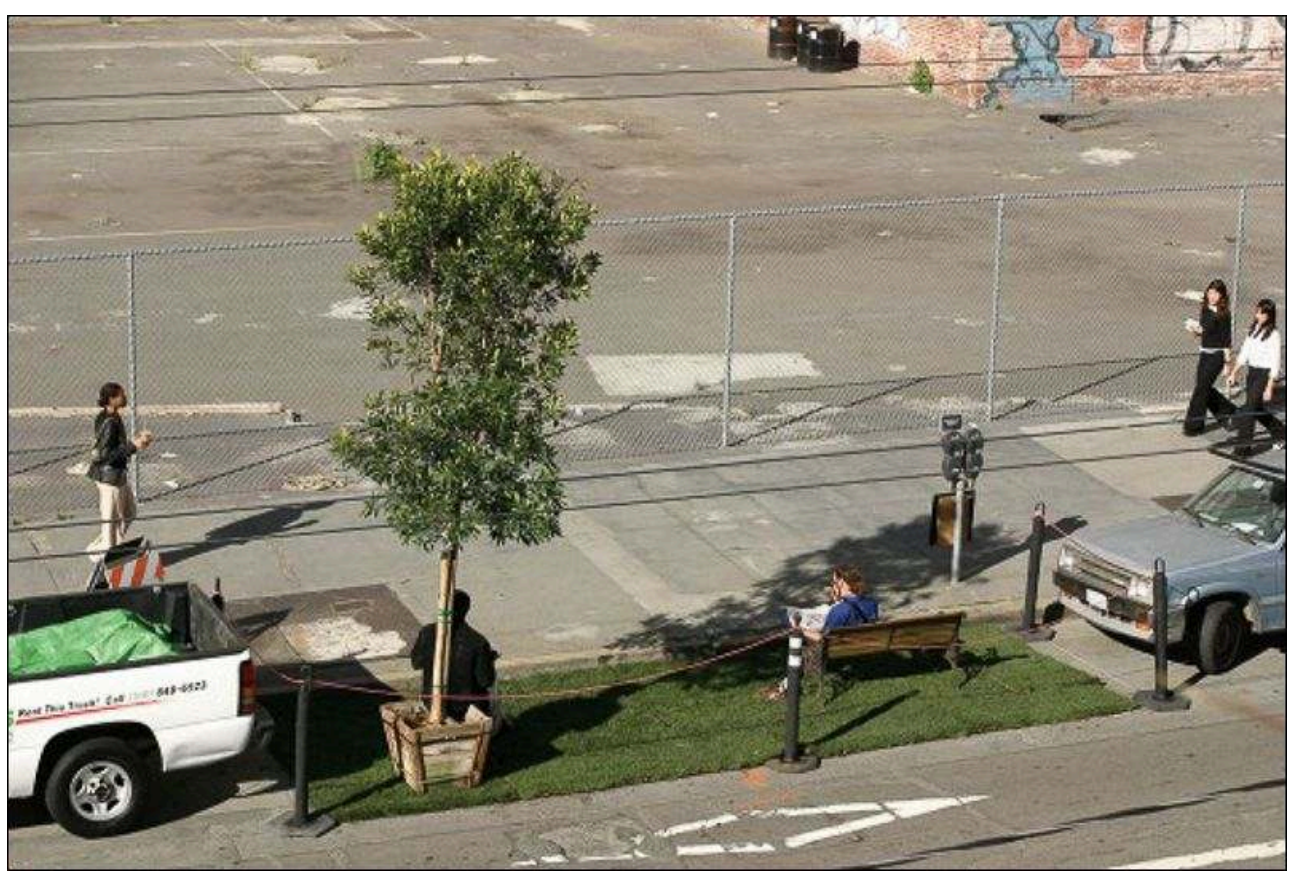

Source : Rebar - licence CC.

Par l'intermédiaire des réseaux sociaux, l'idée s'est progressivement répandue et des actions ont aujourd'hui lieu dans des centaines de villes réparties à travers la planète. En 2008, les activistes du Park(ing) Day revendiquaient plus de 500 installations dans plus de 100 villes à travers l'Amérique du Nord et du Sud, l'Europe et l'Australie. En 2011, ils revendiquaient plus de 975 parcs dans plus de 162 villes réparties dans 35 pays et 6 continents. Park(ing) Day est ainsi devenu un évènement annuel international, à date unique (normalement le troisième vendredi de septembre), pour tous les groupes affiliés au label Park(ing) Day, quel que soit leur pays de résidence (Douay et Prévot, 2014). REBAR se réclame d'ailleurs du mouvement de l'urbanisme tactique qu'il définit comme "the use of modest or temporary revisions to urban space to seed structural environmental change" (Merker, 2010, p. 49) à savoir une action de court-terme, aisément réalisable, aux effets immédiats mais susceptible de changer, à plus long terme, la perception, l'usage et l'attractivité d'un espace à partir d'une dynamique locale. Exactement ce que Myke Lydon théorise la même année comme devant être : « $a$ deliberate phased approach to investigate change ». 


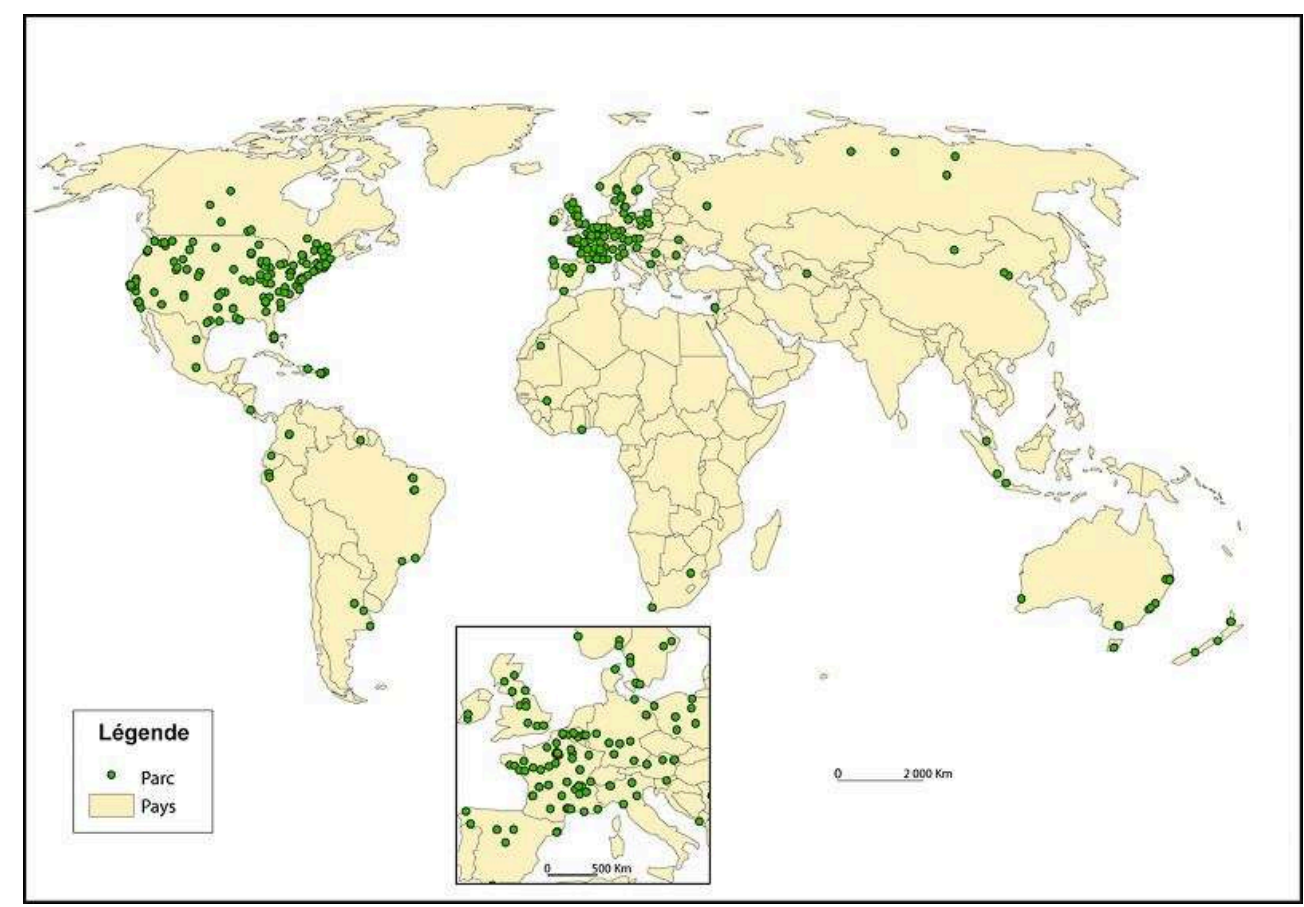

Source : Douay et Prévot, 2014 ; réalisation : Ludovic Chalonge - UMR Géographie-Cités.

Le groupe REBAR présente le Park(ing) Day comme un projet relevant, précisément, de l'open source. Pour autant, chaque individu désireux de rejoindre la communauté et ses actions est enjoint à respecter, moralement et légalement, l'esprit du projet source dont il se réclame, en suivant à la lettre les préconisations exposées dans la licence déposée par Rebar Group inc. Sur le mode d'emploi du Park(ing) Day en libre téléchargement sur son site web, on y trouve toutes sortes de recommandations pour le bon déroulement de l'évènement. Myke Lydon préconise le réalisme comme ligne de conduite. Notons que l'application strictement balisée du label Park(ing) Day, est ainsi souvent déconnectée des enjeux politiques locaux.

\section{Réception et institutionnalisation du tactical urbanism à Paris}

Les idées de l'urbanisme tactique ont évidemment circulé jusqu'à Paris pour venir alimenter toute une série de mobilisations dans la continuité d'initiatives citoyennes comme le Park(ing) Day. Ce modèle semble participer à l'évolution des référentiels de l'action publique municipale qui tend vers une pratique de l'urbanisme à la fois plus durable et plus collaborative. Différents programmes ou opérations illustrent cette institutionnalisation du modèle de l'urbanisme tactique que l'on peut observer à travers une logique descendante (l'événement «Paris-Plage»), ou plus ascendante comme pour l'appel à végétalisation ou les opérations de participation publique. 


\section{L'urbanisme tactique comme pratique institutionnelle pour expérimenter de nouveaux usages de l'espace public : l'exemple de «Paris-Plages »}

L'aménagement des espaces publics donne souvent lieu à des détournements. La présence des bouquinistes sur les quais de la Seine à Paris qui remonte au XVI ${ }^{\mathrm{e}}$ siècle est parfois présentée comme la plus vieille expérience d'urbanisme tactique dans la capitale française $\mathrm{e}^{17}$, avec l'installation de manière réversible de boîtes sur les quais. À partir de 1859, leur métier est légalisé par la Ville de Paris. Abandonnant la voiture à bras, ils ont investi les parapets avec des boîtes vertes dont le format et la couleur sont très réglementés. D'abord cantonnés sur la rive gauche, face aux innombrables libraires des quais Saint-Augustin et Saint-Michel, ils s'étendent vers 1900 sur la rive droite, élargissant la vente de livres d'occasion à des genres littéraires moins nobles (Dupavillon, 2001).

Beaucoup plus récemment, et avec la montée en puissance des enjeux environnementaux, un réseau de militants écologistes s'est engagé dans la transformation de l'espace urbain (Raynaud-Desmet, 2012) en promouvant les initiatives du Park(ing) Day, de la "vélorution », des jardins partagés ou la guérilla gardening.

24 La Ville de Paris va vite suivre une évolution similaire et faire évoluer ses pratiques de l'aménagement urbain, mais dans une perspective plus institutionnelle. Depuis la fin des années 1990, le développement durable est devenu un référentiel de plus en plus dominant qui s'est traduit par un recul de la place dévolue à l'automobile.

L'aménagement des quais de la Seine illustre cette influence de l'urbanisme tactique et du placemaking dans la nouvelle fabrique des espaces publics parisiens. Ceux-ci laissent désormais plus de place aux piétons et aux usages récréatifs. Les interventions, d'abord temporaires, prévoient une réversibilité des usages et se concentrent sur les pratiques et les diverses formes d'appropriations par les citoyens. De 1998 à 2000, la « Fête de la Seine » rend les berges aux piétons pendant un weekend de septembre, le plus souvent en association avec les "journées du patrimoine » et la "journée sans voiture ». En 2001, le premier mandat de Bertrand Delanoë à l'Hôtel de Ville voit la mise en place, chaque dimanche, du dispositif «Paris Respire» qui limite la circulation automobile. Mais c'est surtout la création de l'événement estival annuel « Paris-Plages » qui amorce une réappropriation piétonne plus massive des berges.

Sur plus de 3,5 km de voies sur berges la circulation automobile est ainsi interdite. De même des espaces annexes sont-ils aussi aménagés tels que le parvis de l'Hôtel de Ville ou bien, depuis 2007, le bassin de la Villette. Placé sous la responsabilité de la Délégation générale à l'événementiel et au protocole de la Ville de Paris (DGEP), les installations font l'objet d'une scénographie précise qui s'appuie sur l'installation d'une plage de sable et d'herbe, de palmiers et de mobiliers (voir illustration 5) ad hoc. 


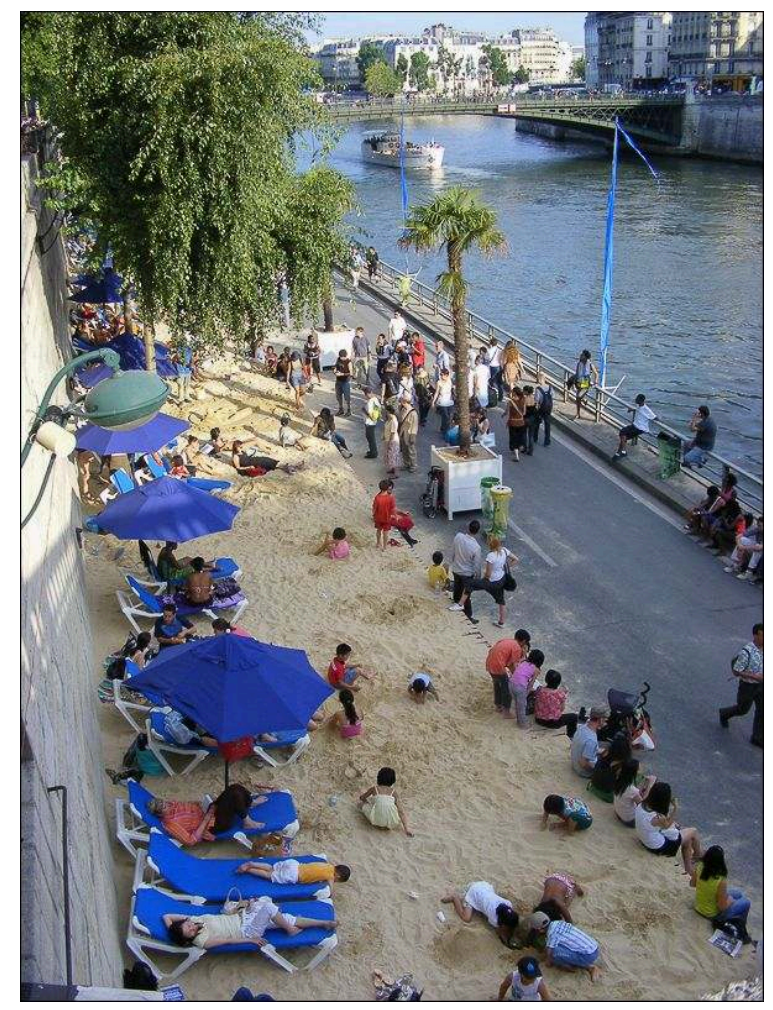

Source : wikipedia - licence CC

Il s'agit donc d'un changement des modes d'action de la municipalité qui développe une pratique moins traditionnelle de l'aménagement:

«Cette évolution illustre une forme d'institutionnalisation de l'aménagement temporaire des espaces publics en tant qu'outil d'urbanisme susceptible d'accompagner des projets urbains en s'intégrant aux différentes étapes de leur réalisation (...) Depuis 2002, Paris Plages a également contribué à préfigurer la transformation prévue d'une partie des berges en boulevard urbain, non seulement en favorisant l'acceptabilité sociale du projet, mais aussi en anticipant les pratiques récréatives et les événements saisonniers qui s'y dérouleront pour stimuler l'animation de cet espace rendu aux piétons. » (Pradel, 2013).

Le succès et la médiatisation de l'événement parisien vont conduire à sa diffusion internationale (à Berlin, Bruxelles, Budapest, Jérusalem ou Montréal) et nationale (Metz, Lille, Tourcoing, Clermont-Ferrand ou encore à Saint-Quentin qui était la pionnière avec une mise en place dès 1996).

\section{Urbanisme tactique et Big Data comme pratique institutionnelle pour repenser l'espace public : le réaménagement de la place de la Nation}

29 L'urbanisme tactique peut aussi s'envisager en amont des projets urbains. Dans cette perspective, l'apport des pratiques citoyennes est mobilisé dans une démarche d'expérimentation puis d'évaluation.

C'est le cas du réaménagement de la place de la Nation. L'originalité de la démarche, officiellement présentée comme relevant de l'urbanisme tactique, est d'utiliser des données massives (Big Data) d'observation des usages de l'espace public captées par des 
dispositifs numériques à même de fournir une connaissance nouvelle :« Nous offrons à la ville de Paris l'opportunité de mettre en place une politique de tactical urbanism, c'est-à-dire de changement progressif, ponctuel et réversible grâce à des expérimentations sur des points précis pour lesquelles nous sommes capables de fournir des informations fiables sur leur efficacité avant d'envisager leur généralisation" affirme Martin Lagache, cadre de l'entreprise Placemeter ${ }^{18}$, fondée en 2012 et originellement localisée à New York, qui poursuit : «Le tactical urbanism y est utilisé depuis plusieurs années pour redynamiser le centre-ville et notamment à Times Square, où la piétonisation a d'abord été testée par intermittence entre 2009 et 2014, pour en mesurer les conséquences, avant de l'adopter définitivement ».

31 Les données servent à identifier les usages puis à évaluer les appropriations des aménagements afin de permettre une certaine réversibilité et adaptation:

"La clé, c'est l'évaluation. Avec ces données, nous pourrons appuyer les projets d'aménagement de la ville et aider à la décision. Nous pourrons prévoir, par exemple, l'élargissement d'un trottoir en fonction du flux de piétons, adapter les voies de circulation en fonction des types de véhicules qui traversent le plus la place ou adapter le mobilier urbain selon les besoins" (Benjamin Favriau responsable de projets dans la mission «Ville intelligente et durable » de la mairie de Paris) ${ }^{19}$.

L'importation, par la Ville de Paris, de cette plate-forme américaine de recueil de données numériques appliquée à la résolution de problématiques d'aménagement et d'usages urbains est un autre exemple des phénomènes de circulation et d'importation évoqués précédemment. Néanmoins le lien ici fait entre une institution (La Ville de Paris), les Big Data et l'urbanisme tactique doit être interrogé. La ville intelligente, ou "smart city", serait ainsi devenue l'alliée "naturelle» de l'urbanisme tactique, une nouvelle façon de penser la ville, le service urbain et l'interaction de ses différents acteurs (administration, habitants, entreprises). Métropolisation, complexité croissante du fait urbain, place accrue du numérique et des données, nécessité de contenir la dérive des dépenses publiques locales, la smart city alliée à/de l'urbanisme tactique serait-elle à la fois le lieu et le moyen de relever les défis de l'urbanisation et des opportunités et d'y faire face ${ }^{20}$ de manière ici plutôt descendante de la part des pouvoirs publics?

\section{L'urbanisme tactique comme pratique participative : l'appel à projet de végétalisation}

L'urbanisme tactique, au départ, n'est précisément pas une démarche descendante des pouvoirs publics mais s'inscrit dans une perspective ascendante visant à mobiliser les citoyens via des dispositifs spontanés ou participatifs. Ces derniers connaissent un développement important dans les villes occidentales venant ainsi illustrer le tournant vers un urbanisme plus collaboratif. Dans un contexte où les ressources budgétaires se contractent, le recours à des logiques d'urbanisme tactique par les pouvoirs publics permettrait en effet de répondre à une demande participative de manière formelle, autour d'un projet concret de transformation de l'espace, alors même que de nombreux appels participatifs à projet auraient pour mérite et vertu de renforcer l'acceptabilité sociale des politiques publiques tout en reportant une partie des coûts de la mise en œuvre et de l'entretien de ces projets sur les citoyens eux-mêmes.

35 À Paris, une telle dynamique peut être observée, autour des appels à projets de végétalisation notamment, institutionnalisant ainsi des expériences antérieures 
autogérées telles que celle d'Ecobox dans le quartier de la Chapelle ${ }^{21}$, en cours depuis 2001, et emboitant aussi le pas à d'autres initiatives américaines comme le 45 Lansing Street à San Francisco ${ }^{22}$, ou européennes, à l'image de Stalled Place itiniatives à Glasgow $^{23}$ en 2010 et du Plan Buits à Barcelone (Orduna-Giro P. Jacquot S., 2014) en 2011. En 2014, en effet, la Mairie de Paris lance le programme « Du vert près de chez moi $~^{24}$ et propose aux citoyens de recenser les lieux susceptibles d'être végétalisés : murs, trottoirs, placettes ou encore façades, et ce grâce à différents dispositifs, notamment numériques avec la mise en place d'application et de carte participative ${ }^{25}$. Selon Pénélope Komitès, adjointe chargée des espaces verts, «le but de ce projet est de créer un nouveau modèle urbain, où la place de la nature en ville n'est pas seulement dans les parcs, les jardins et les bois, mais partout dans l'espace public ». Ce dispositif vient institutionnaliser des mobilisations sociales environnementales qui ont la ville comme objet et comme terrain (Hou 2000; Douay et Prévot, 2014), à Paris comme ailleurs. Deux modes de réalisation sont proposés par la Ville. Lorsque l'aménagement concerne un espace public, la Mairie peut prendre en charge la réalisation et l'entretien de jardinières de pleine terre, de murs végétalisés ou de plantations d'arbres. La démarche participative permet alors une définition de la politique publique au plus près des citoyens. Par ailleurs, la Ville peut aussi réaliser l'aménagement ou fournir le mobilier avant que les riverains prennent en charge l'entretien de ces espaces, à l'image des 96 jardins partagés de Paris (Demailly, 2014). Les habitants s'engagent ici à entretenir ces espaces collectifs végétalisés en coordination avec les services de la Ville. Après plus de deux mois de consultation, 1500 lieux ont été proposés par les habitants et 209 ont été sélectionnés (voir illustration 6). Les projets sont répartis sur l'ensemble du territoire parisien à l'exception des arrondissements les plus chics $\left(6^{\mathrm{e}}, 7^{\mathrm{e}}, 8^{\mathrm{e}}, 16^{\mathrm{e}}\right.$ et en partie pour les $15^{\mathrm{e}}$ et $\left.17^{\mathrm{e}}\right)$. Cette concentration dans les arrondissements populaires ou gentrifiés donne une indication sur le profil social des citoyens ou groupes qui vont répondre à ces appels. La localisation des parcs du Park(ing) Day (Douay et Prévot, 2014, p. 30) suit, elle aussi, en partie, les mêmes logiques en se concentrant dans les arrondissements du Nord-Est parisien qui constituent le cœur des espaces gentrifiés de la capitale (voir illustration 6). Dans ce sens, l'engagement en faveur de l'urbanisme tactique dans ces espaces gentrifiés semble participer de la dynamique locale de "branding ». Les anglosaxons la désignent sous le vocable d' "urban vibrancy», qui se traduit par une "cleansing gentrification ${ }^{26}$ et produit une exclusion des classes populaires de ces quartiers. 
Illustration 6 - Localisation des parcs du Park(ing) Day et des projets de végétalisation sélectionnés

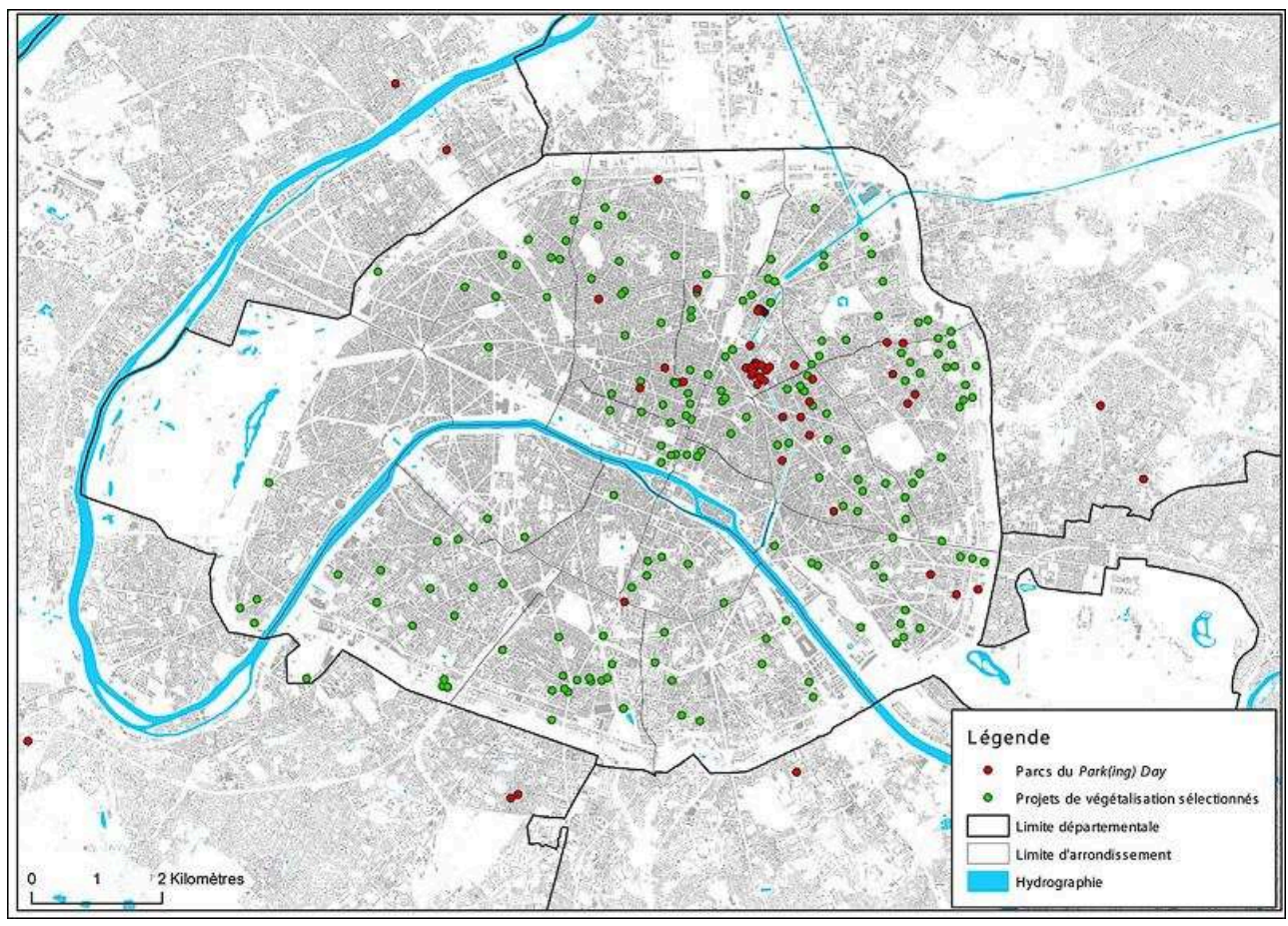

Source : données de l'édition 2013 du Park(ing) Day: http://parkingday.org ; données 2015 de la Mairie de Paris : http://opendata.paris.fr/map/embed/

du_vert_pres_d_echez_moi_les_209_propositions_selectionnees/

La plupart du temps les installations sont assez simples avec des pots ou des bacs de fleurs et plus rarement la plantation d'arbres (voir illustration 7). C'est une illustration de ce qu'est l'urbanisme tactique du «Do it Yourself»: la réponse simple, rapide, concrète, peu coûteuse aux demandes exprimées localement par les citoyens 


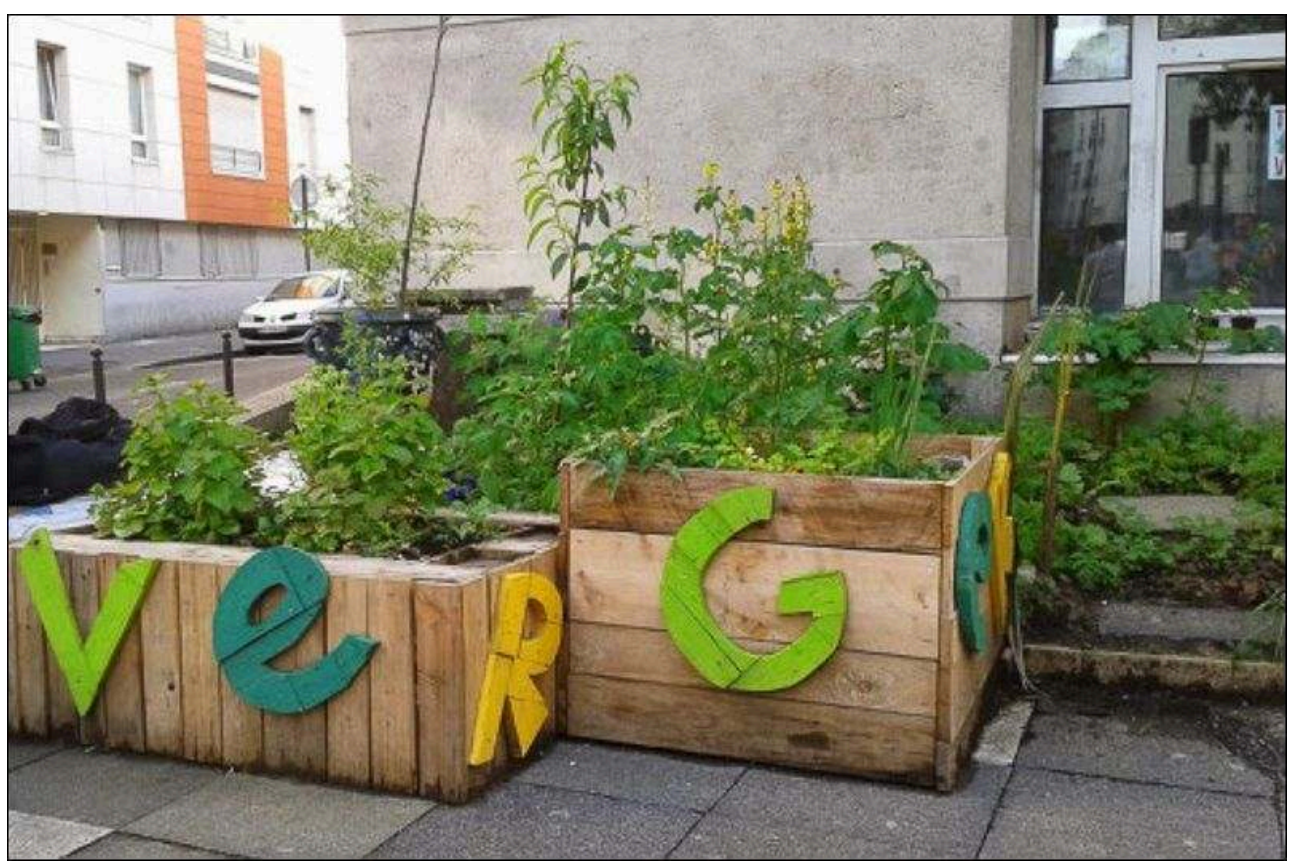

Source : http://acteursduparisdurable.fr/actus/du-vert-pres-de-chez-moi

Depuis juin 2015, un «permis de végétaliser " permet l'extension de la démarche pour l'ensemble des projets qui concernent la voie publique. Les citoyens peuvent être accompagnés en recevant un kit de plantation (graines et terre végétale). Ils doivent s'engager à utiliser des plantes locales et à favoriser la biodiversité, à ne pas recourir à des pesticides et à veiller à l'esthétique et à l'entretien des plantes et supports ${ }^{27}$. La nouvelle offre participative de la Ville de Paris en faveur de la végétalisation est ici censée répondre au désir croissant de «nature en ville». Elle offre une alternative semble-t-il plus attrayante et mobilisatrice que les dispositifs participatifs moribonds que sont les conseils de quartiers qui peinent à se renouveler. Comme dans le cas barcelonais, on peut aussi y voir une intention d'ordre gestionnaire ou managérial : fabriquer des citoyens plus "responsables", tout en encourageant le lien social. Il ne s'agit pas seulement de demander aux habitants ce qu'ils veulent, mais de les inciter à faire eux-mêmes. Plus généralement, cette gamme d'initiatives doit aussi nous interroger sur la sélection des usages qui est ainsi faite (mobilité, loisirs, nature, commerce). Cette sélection signifie-t-elle que certaines activités de la ville seraient désavouées dans ces espaces? Ces nouveaux usages prescrits ne seraient-ils pas socialement situés, à partir des pratiques de certains groupes sociaux (embourgeoisement, résidentialisation d'un quartier, etc.)? Ces interrogations soulevées par Benjamin Pradel semblent trouver un début de réponse dans cet exemple parisien.

\section{Du modèle alternatif au nouveau mainstream ?}

La popularité croissante de l'urbanisme tactique - avec la constitution d'un réseau militant actif socialement et culturellement homogène -, et son institutionnalisation 
rapide questionnent le devenir de son caractère originellement alternatif : n'assisterait-on pas en réalité à l'émergence d'un nouveau modèle dominant?

\section{Détourner par la collaboration}

39 La dimension systématiquement ludique, colorée, et conviviale des installations de ces collectifs peut être envisagée comme un pied de nez à la morosité ambiante d'un avenir individuel et collectif pris dans les remous - si ce n'est la nasse - de la mondialisation avec son cortège de crash économique, de terrorisme, de déclassement social, de désaffiliation sociale. Ce serait une manière de dresser, avec trois fois rien comme moyen : «l'étendard des échanges désintéressés et des valeurs du don promus par la génération des pionniers du Net » (Dagnaud, 2013, p.. 104-105) pour reconfigurer, à la manière des Indignés et des Nuits Debout, l'espace, non seulement urbain, mais plus profondément et symboliquement délibératif. Ainsi le mouvement Nuit Debout a-t-il installé un amphithéâtre et une salle de cinéma à partir d'une sortie de métro qui était condamnée ${ }^{28}$; une installation bien sûr assez légère et réversible.

Illustration 8 - L'aménagement d'un amphithéâtre par le mouvement Nuit Debout

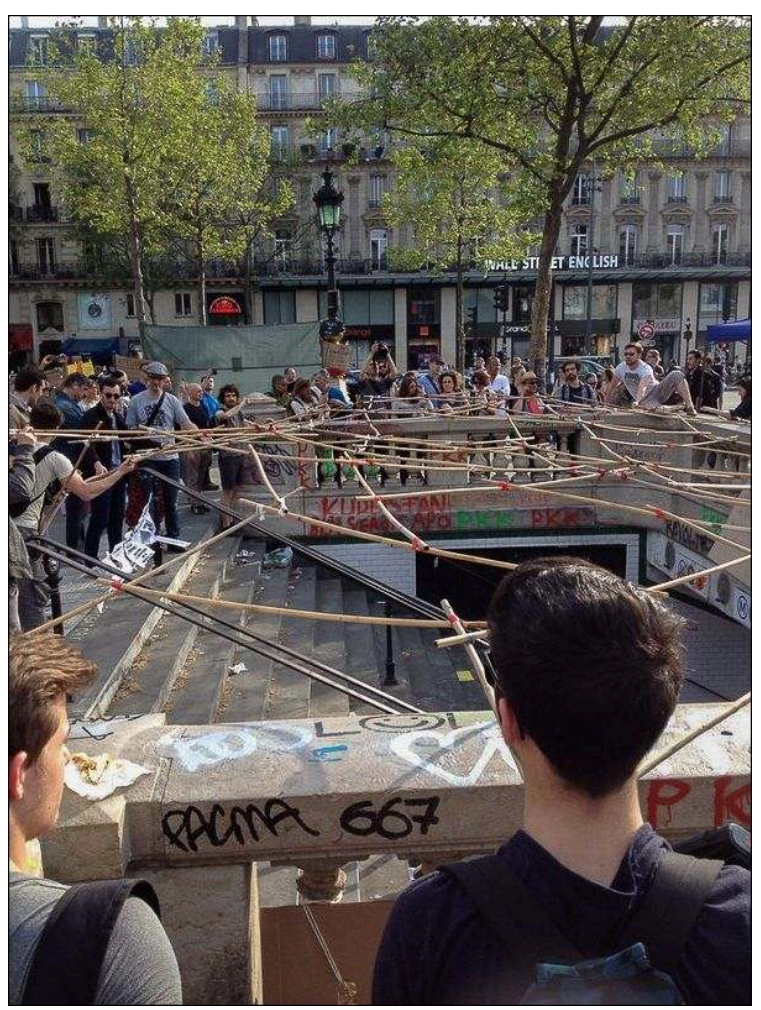

Source :@LarrereMathilde ; https://twitter.com/LarrereMathilde/status/729347909603495936/photo/ 1 


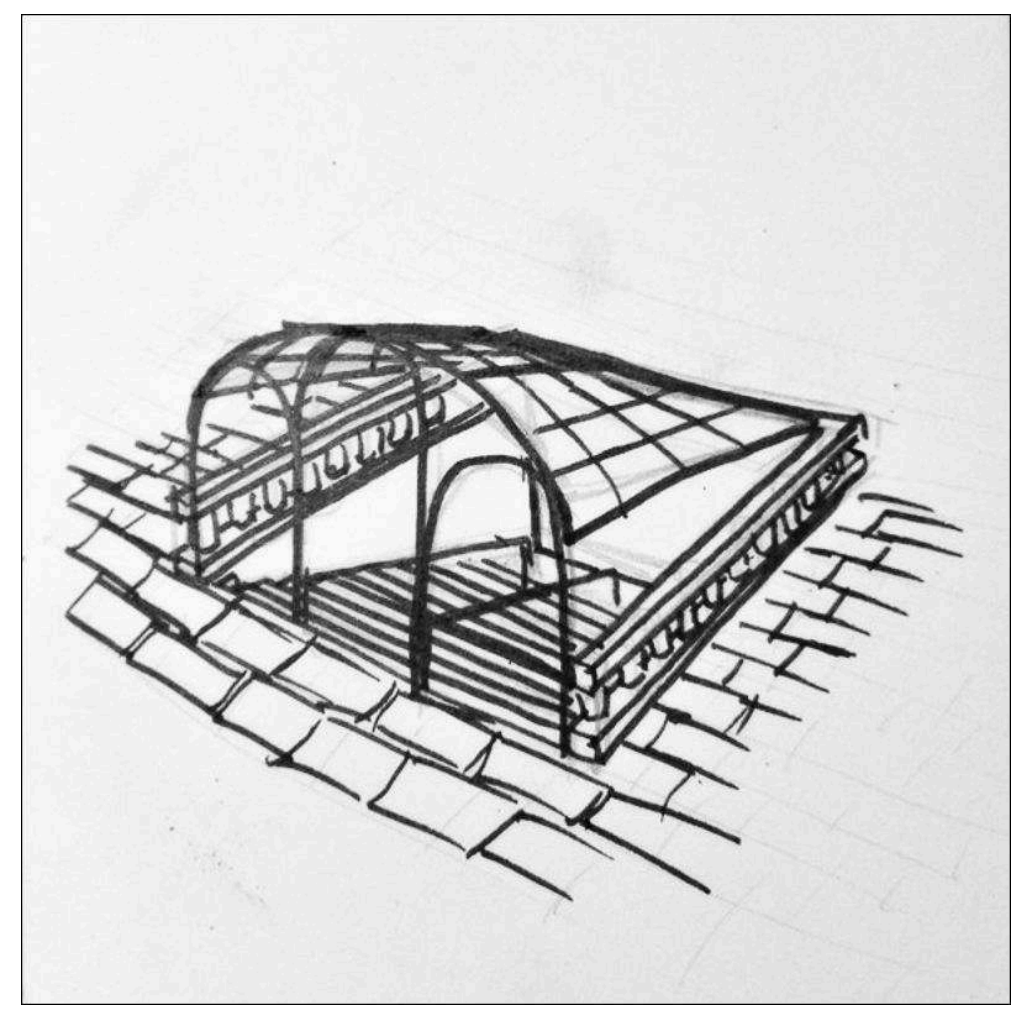

Source : @Anachitect ; https://twitter.com/Anachitect/status/729253374554738689/photo/1

Du détournement des usages ordinaire d'un lieu au détournement de sens entrant résolument dans le champ politique il n'y a qu'un pas, franchi au moment où les anciennes postures et figures politiques paraissent de plus en plus déconnectées de la réalité quotidienne des individus et sont accusées d'être somme toute assez peu habitées par une conception haute de l'intérêt général. Ces initiatives bricolées sont, à leur manière, des micro-antidotes au désenchantement et au scepticisme résigné de la génération de Mai 68.

\section{Un réseau homogène et qui tend à se professionnaliser}

41 L'enquête menée par Gordon Douglas (2014), sur ceux qu'ils nomme les « Do It Yourself urbanists " montre d'abord que les créateurs, contributeurs ou activistes urbains investis sous ce vocable appartiennent précisément à une jeunesse urbanophile, connectée et créative réinvestissant des centre-villes longtemps désertés et d'autres quartiers laissés en désuétude, que ce soit en Amérique du Nord ou en Europe. La localisation des installations du Parking Day ou des projets de végétalisation à Paris (voir illustration 6) montre bien ce phénomène. Cette génération dite des millenials, ces jeunes nés entre les années 1980 et 2000, est également connue sous le nom de génération Y (Dagnaud, 2013). Elle partage le sentiment de posséder le capital social et culturel pour régler les problèmes dans les espaces urbains qu'elle pratique et l'urbanisme tactique lui en a fourni les modalités pratiques et les occasions.

Dans ce sens, l'urbanisme tactique tel qu'il a pu être observé sur le terrain illustrerait plutôt les évolutions contemporaines des mobilisations urbaines. Les mouvements sociaux classiques laissent ainsi place à des revendications idéologiquement et 
doctrinalement moins radicales et plus diverses, marquées par le passage de revendications matérialistes, essentiellement liées aux conditions de travail (caractéristiques du mouvement ouvrier), à des préoccupations post-matérialistes (environnement/cadre de vie). Ainsi, l'identité du ou des groupe(s) militant(s) s'en trouve-t-elle modifiée, son fondement étant désormais extérieur au monde du travail. Le critère déterminant ne serait désormais plus la classe à laquelle on appartient, mais le style de vie que l'on a adopté ou d'autres appartenances (sexuelles, raciales, géographiques, etc.). Le fonctionnement des groupes militants se renouvelle aussi, rejetant les organisations traditionnelles (syndicats ou fédérations habitantes) au profit d'organisations plus décentralisées marquant leur souhait d'autonomie et finalement porteuses de plus d'incertitude quant aux formes urbaines à produire. Il ne s'agit plus de remettre en cause l'organisation de la société en rejetant, par exemple, le modèle néo-capitaliste. L'urbanisme tactique ne se pose pas en s'opposant à la planification urbaine institutionnelle et à l'urbanisme de projet. Il s'insère dans un espace laissé vide par les failles d'un système qui néglige par trop souvent l'échelle de la vie quotidienne. La dimension politique et possiblement conflictuelle s'efface au profit de l'évènementiel et $\mathrm{du}$ divertissement qui permet de fédérer des communautés partageant la même vision du monde et de la ville et un mode de vie similaire. Dans L'inflation créatrice, Albert Meister (1975) relevait cette tendance à la «fête permanente ", à l'évasion, à la distraction, à la culture distillée et l'évènement réitéré, bref, à l'intégration généralisée portée par des classes moyennes urbaines conjuguant capital social et capital scolaire. Cet investissement des classes moyennes urbaines dans la résolution des problématiques d'aménagement et de cadre de vie n'est pas nouveau. Dans les années 1960 et 1970, un certain nombre de militants issus des classes moyennes urbaines ont alors conjugué engagement politique (PSU), syndical ou associatif avec une carrière professionnelle dans les structures d'études urbaines parapubliques ou privées (Prévot, 2015) ou dans l'opérationnel. Si le contexte, les objectifs et les modes de faire ont bel et bien changé depuis les Trente Glorieuses, une tendance certaine à la professionnalisation des activistes est aujourd'hui observable. Elle va bien souvent de pair avec une coopération entre ces derniers et les pouvoirs publics, prélude le plus souvent à une institutionnalisation des dispositifs. L'agence Dédale qui ne vit quasiment que de subventions publiques pour la réalisation de projets d'animation socio-culturelle en est un exemple. Par ailleurs, la plupart des figures américaines de l'urbanisme tactique exercent aujourd'hui comme consultants en design urbain et proposent leurs services aux institutions intéressées. Dès lors, dans quelle mesure l'urbanisme tactique est-il toujours un modèle alternatif ou bien n'est-il devenu qu'une énième variante ou variable d'ajustement du modèle dominant?

\section{Une nouvelle temporalité urbaine placée sous le signe de l'austérité budgétaire?}

43 Avec la «ville malléable », Luc Gwiazdzinski et Jérôme Massiani (2015) ont proposé un concept transversal qui place la question des temps au centre de l'organisation de la vie urbaine. L'idée est de proposer une polyvalence de l'espace, en particulier par des usages alternés. De manière générale, le temps illustre la tension à laquelle sont soumises les collectivités dans le contexte néolibéral actuel, entre stratégies d'attractivité et garantie de l'intérêt général. 

gestion transitoire d'espaces urbains en friche pour en détourner les usages existants ou pour en suggérer d'autres de manière éphémère. Toutes ces actions sont placées sous le sceau de l"austérité budgétaire et du désengagement de l'État. Les municipalités citées dans cette contribution ont toutes privilégié un aménagement à moindre coût, par la cession temporaire et gratuite d'un espace public, pour un usage non lucratif, à une entité, associative le plus souvent, et parfois inscrite dans une logique de mécénat ${ }^{29}$. Benjamin Pradel (2015) a identifié quatre formes d'urbanisme temporaires, selon les types d'acteurs impliqués et les objectifs visés par les projets: les espaces publics illégaux, les espaces réversibles, l'urbanisme tactique et l'urbanisme événementiel. En citant parmi les conditions de leur émergence le temps de plus en plus court de l'action publique ou l'urgence des mandats électoraux, il a oublié, nous semble-t-il, l'austérité budgétaire généralisée. Ces initiatives doivent aussi être envisagées d'un point de vue économique. Ces interventions éphémères ou transitoires permettent en effet aussi à ces sites, pendant cet entre-temps de crise que la conjoncture peut allonger, de ne pas perdre ou de regagner de la valeur, en partie symbolique seulement. Ces interventions constituent une alternative à l'abandon qui, lui-même, a un coût et à un réinvestissement immédiat impossible du fait des délais parfois longs d'un aménagement. Ce faisant, ces divers types d'initiatives, lorsqu'elles sont approuvées voire encouragées par les autorités ou les gestionnaires de ces sites visent aussi à baisser les coûts de mise en sécurité et d'entretien et à « influencer la valeur marchande d'un espace et encourager son redéveloppement à court ou moyen terme » (Andres, 2011), le tout dans une économie de moyens pour tous.

\section{Vers un nouveau modèle dominant}

Aujourd'hui, les institutions en charge de l'aménagement se sont bel et bien appropriées le langage et les codes de cet urbanisme tactique, et ce d'autant plus aisément que les manuels ou autres modes d'emplois diffusés en accès libre par les figures de l'urbanisme tactique, ont eux-mêmes normalisé et... codifié des actions originellement spontanées et illégales pour les « labelliser » et les dupliquer à travers le monde. Tous insistent avant tout sur leur caractère événementiel, leur portée symbolique. Elles permettraient d'illustrer un possible, sans jamais prétendre le réaliser pour de bon. Celles-ci doivent en outre impérativement se soumettre aux lois et règlements en vigueur sur le site de l'installation ce qui vient grandement limiter toute perspective conflictuelle et toute idée de participation agonistique.

L'urbanisme tactique est un nouvel élément essentiel des politiques urbaines contemporaines de métropoles qui se trouvent prises dans des logiques de compétition pour attirer les investissements, les créateurs et les touristes. Ainsi l'urbanisme tactique fait-il désormais partie d'un langage urbain assez attendu qui participe aux logiques de standardisation et d'homogénéisation des politiques urbaines. L'adoption de ce code par le plus grand nombre en réduit la dimension réellement alternative. Si le principe de licence ouverte remet en cause le schéma du capitalisme en établissant une coopération horizontale entre les acteurs et en faisant éclater les systèmes propriétaires, l'urbanisme tactique semble avoir, au moins en partie, perdu tout caractère transgressif pour devenir, à l'instar du Park(ing) Day une marque franchisée/ label (Douay et Prévot, 2014), voire une méthode à la mode de valorisation économique de projets. Les festivals dédiés en sont un autre exemple, à l'image du Festival Village

EchoGéo, 36 | 2016 
Building Convergence $e^{30}$ mis en place à la suite du succès rencontré par les Intersection Repair à Portland autour de l'initiative de Mark Lakeman qui, en 1996, avait décidé de mobiliser les habitants de son quartier pour repeindre de manière fantaisiste et colorée une intersection routière et $\mathrm{y}$ installer jeux pour enfants et mobilier urbain. Les autorités de la ville avaient rapidement soutenu l'action par un décret municipal autorisant officiellement les habitants à développer librement d'autres espaces de ce genre. L'urbanisme tactique semble ainsi désormais incorporé, voire digéré, dans/par les stratégies urbaines de production de la ville dite « créative » néo-libérale.

\section{Conclusion : l'ambiguïté d'une rébellion}

Le mouvement de l'urbanisme tactique est formellement apparu en Amérique du Nord avant de se diffuser en Europe et dans le reste du monde, principalement occidental. À l'image d'autres politiques territoriales qui vont circuler dans l'espace les villes jouent ici aussi une place singulière. Les échanges s'appuient sur une communauté d'esprit, de pratiques et d'expertises aux frontières mouvantes. En effet, le recours aux ressources numériques permet une mise en réseau fluide et ouverte à l'échelle internationale mais peu hiérarchisée. La formalisation de ces pratiques passe surtout par une institutionnalisation locale de celles-ci qui viennent renouveler les références des politiques publiques d'aménagement urbain.

L'étude du cas de Paris a montré la réception de ces pratiques, localement, par un ensemble de citoyens ou de groupes organisés qui ont pu développer des pratiques s'inspirant de l'urbanisme tactique, à l'image du mouvement Park(ing) Day. Cependant, le caractère réellement "alternatif» de ces pratiques n'est pas avéré, l'urbanisme tactique ne change pas substantiellement la nature des politiques urbaines et des rapports de pouvoirs. Il s'agit plutôt d'une nouvelle attention portée aux espaces publics et à ses appropriations citoyennes en temps de crise et d'austérité budgétaire. Ainsi, les institutions elles-mêmes s'approprient ce vocabulaire afin de mieux mettre en scène leurs actions. L'urbanisme tactique est donc devenu un élément essentiel d'un urbanisme mainstream, celui d'une ville néo-libérale qui se doit d'être créative et participative. Mais il peut aussi être envisagé comme le levier ou le vecteur d'une démocratie "impliquante $"^{31}$ dans laquelle les habitants ne se penseraient pas comme sujets-soumis consommateurs de l'action publique mais des sujets-acteurs coproducteurs de l'intérêt général.

\section{BIBLIOGRAPHY}

Ardenne P., 2002. Un art contextuel, création artistique en milieu urbain, en situation d'intervention, de participation. Paris, Flammarion.

Bacqué M-H., Biewener C., 2013. L'empowerment, une pratique émancipatrice ? Paris, La Découverte. 
Béal V., Rousseau, M., 2008. Néolibéraliser la ville fordiste. Politiques urbaines post-keynésiennes et re-développement économique au Royaume-Uni : une approche comparative. Métropoles, $\mathrm{n}^{\circ} 4$, p. 160-202.

Brenner N., Theodore N. (eds.), 2002. Spaces of Neoliberalism: Urban Restructuring in North America and Western Europe. Oxford, Blackwell.

Bigand S., 2010. Regards sur la densité urbaine. Mémoire de stage de master 2 Aménagement, Urbanisme, Développement des Territoires (AUDT), Université de Lille 1, dir. Prévot M. (tuteur universitaire) et De la Gorce D. (agence Pattou-Tandem).

Capelli C., 2013. Expérimenter pour faire la ville “durablement” Exploration du cas de l'urbanisme tactique aux États-Unis. Master Sciences du territoire, Institut d'Urbanisme de Grenoble.

Casagrande M., 2010. Urban Acupuncture. Adam Parsons, University of Portsmouth.

Certeau (de) M., 1990. L'invention du quotidien. Paris, Folio.

Crombez R., 2014. La ville sans les urbanistes? Urbanisme tactique en Amérique du Nord. Une étude de cas Articiste à Toronto. Mémoire de stage de master 2 Aménagement, Urbanisme, Développement des Territoires (AUDT), Université de Lille 1, dir. Prévot M. (tuteur universitaire) et Jianfar M. (STPES Iniatiative).

Dagnaud M. 2010. Le web, ce laboratoire du capitalisme sympa. Le Débat, $\mathrm{n}^{\circ} 160$.

Dagnaud M., 2013. Génération Y. Les jeunes et les réseaux sociaux, de la dérision à la subversion. Paris, Presses de Sciences Po, coll. Nouveaux Débats.

Denais M., 2012. Activisme urbain : Park(ing) Day et le projet 8m2, deux formes de réappropriation citoyenne des places de stationnement. Mémoire de stage de master 2 Aménagement, Urbanisme, Développement des Territoires (AUDT), Université de Lille 1, dir. Grégoris M.-T. et Prévot M..

Dolowitz D., Marsh D., 1996. Who Learns What from Whom: A Review of the Policy Transfer Literature. Political Studies, $n^{\circ}$ 44(2), p. 343-357.

Dolowitz, D., Marsh, D., 2000. Learning from Abroad: the role of policy transfer in contemporary policy-making. Governance, 13 (1), p. 5-24.

Douay N., Duaso I., 2008. Glamour planning ou les villes à l'ère de la competition. In N. Buchoud dir, La ville stratégique Changer l'urbanisme pour répondre aux défis urbains mondiaux, Lyon, Éditions du CERTU, p. 145-153.

Douay N., Prévot M., 2012 (eds). L'information géographique, n " L'activisme urbain : art, architecture et espace public ». Paris, Armand Colin, Vol. 76.

Douay N., Prévot M., 2014. Park(ing) Day : label international d'un activisme urbain édulcoré. Environnement urbain, Dossier thématique : Les arts : révéler, critiquer et transformer les rapports entre individus, environnement et ville, p. 14-33.

Douglas G., 2014. Do-it-Yourself Urban Design: The Social Practice of Informal "Improvement" Through Unauthorized Alteration. City \& Community, vol. 13, n 1, p. 5-25.

Dupavillon C., 2001. Paris côté Seine. Paris, Le Seuil.

Duval A., 2014. L'usage d'internet dans les pratiques de la démocratie participative en aménagement, l'exemple du site internet Strasbourg 2028. Mémoire de master 1 Aménagement et développement local, Université Paris-Diderot, dir. Douay N.

Espaces et Sociétés, 2007. Villes et 'best practices'. n 131. 
Finn D., 2014. DIY urbanism: Implication for cities. Journal of Urbanism, 7(4), p. 381-398.

Gravari-Barbas M., Jacquot S., 2007. L'événement, outil de légitimation de projets urbains : l'instrumentalisation des espaces et des temporalités événementiels à Lille et à Gênes. Géocarrefour, Vol. 82/3 | 2007, mis en ligne le 01 octobre 2010. http://geocarrefour.revues.org/ 2217

Gwiazdzinski L., Massiani M., 2015. Penser la ville malléable : vers une approche spatio-temporelle des politiques urbaines. Collectif d'Action pour la Ville/Expérimentation et Réflexion In situ (COLAVERI) http://colaveri.com/penser-la-ville-malleable-vers-une-nouvelle-approche-spatiotemporelle-des-politiques-urbaines-luc-gwiazdzinski-et-marie-massiani

Hall T., Hubbard P. (eds.), 1998. The Entrepreneurial City: Geographies of Politics, Regime, and Representation. Chichester et New York, Wiley.

Harvey D., 1989. From Managerialismto Entrepreneurialism: The Transformation in Urban Governance in Late Capitalism. Geografiska Annaler B, vol. 71, n 1, p. 3-17.

Hayat S., 2013. Démocratie agonistique. Dictionnaire critique et interdisciplinaire de la participation, Paris, GIS Démocratie et Participation, URL : http://www.participation-et-democratie.fr/node/ 1306

Hou J., 2010. Insurgent Public Space: Guerrilla Urbanism and the Re-Making of Contemporary Cities. New York, Routledge.

Ion J., 1997. La fin des militants ? Paris, L'Atelier.

Iveson K., 2013. Cities within the city: Do it yourself urbanism and the right to the city. International Journal of Urban and Regional Research, 37(3), p. 941-946.

Jacobs J., 1961. Death and life of great American Cities. New York, Random House.

Jolé M., Tonnelat S., 2010. "Placemaking": a new approach to designing and managing urban public spaces. An interview with Kathy Madden and Fred Kent. Metropolitics, 23 November 2010. http://www.metropolitiques.eu/Placemaking-a-new-approach-to.html

Knops A., 2007. Debate: Agonism as Deliberation - On Mouffe's Theory of Democracy. Journal of Political Philosophy, 15(1), p. 115-126.

Latour B., 2006. Changer de société - Refaire de la sociologie. Paris, La Découverte (éd. originale, 2005, Re-Assembling the Social. A Introduction to Actor-Network Theory, Oxford : Oxford University Press).

Lefevre H., 1968. Le droit à la ville. Paris, Anthropos.

Lefebvre H., 1974. La production de l'espace. Paris, Anthropos.

Lerner J., 2007. Acupuncture urbaine. Paris, L'Harmattan, coll. Villes et entreprises, préface d'A. Voisin.

Lydon M., 2011. Tactical Urbanism, Short-Term Action, Long-Term Change. Vol. 1. New York, The Street Plans Collaborative.

Lydon M., Garcia A., 2015. Tactical Urbanism: Short-Term Actions for Long-Term Change. Washington DC, IslandPress.

Manovitch L., 2001. The Langage of New Media. Cambridge: MIT Press.

Masboungi A., 2001. Bilbao : la culture comme projet de ville. Paris, Documentation Française - coll. Projet urbain. 
McCann E., 2011. Urban policy mobilities and global circuits of knowledge. Towards a research agenda. Annals of the Association of American Geographers, $n^{\circ} 101(1)$, p. 107-130.

McCann E., Ward K., 2011. Mobile Urbanism: City policymaking in the global age. Minnesota, University of Minnesota Press.

McCann E., Ward K., 2012. Assembling urbanism: Following policies and 'studying through' the sites and situations of policy making. Environment and Planning A, vol. 44, p. 42-51.

Meister A., 1975. L'inflation créatrice. Paris, PUF.

Merker B., 2010. Taking place: rebar's absurd tactics in generous urbanism. In Hou J. dir., Insurgent Public Space. Guerilla Urbanism and the Remaking of Contemporary Cities, Routledge New York, Routledge. p. 45-58.

Mouffe C., 2000. For an agonistic model of democracy. The Democratic paradox, London, Verso, p. 80-107.

Moulaert F., Rodriguez A., Swyngedouw E. (Eds.), 2005. The Globalized City: Economic Restructuring and Social Polarization in European Cities. Oxford, Oxford University Press.

Mould O., 2014. Tactical Urbanism: The New Vernacular of the Creative City. Geography Compass, Vol. 8, No. 8, p. 529.

Orduna-Giro P., Jacquot S., 2014. La production participative d'espaces publics temporaires en temps de crise. Le projet "Pla Buits" Barcelone. Métropolitiques http://www.metropolitiques.eu/ La-production-participative-d.html

Offerlé M., 1996. Sociologie des groupes d'intérêt. Paris, Montchrestien.

Pradel B., 2013. Sous les pavés, Paris Plages. Métropolitiques, 8 juillet 2013. URL : http:// www.metropolitiques.eu/Sous-les-paves-Paris-Plages.html

Pradel B., Goelzer S., 2015. Quand les citoyens participent à la fabrique de la ville : l'exemple des espaces publics temporaires. Collectif d'Action pour la Ville/Expérimentation et Réflexion In situ (COLAVERI) http://colaveri.com/quand-les-citoyens-participent-a-la-fabrique-de-la-villelexemple-des-espaces-publics-temporaires-benjamin-pradel-et-sebastien-goelzer

Peck J., Theodore N., 2010. Mobilizing Policy: Models, Methods, and Mutations. Geoforum, n 41, p. 169-174.

Peyroux E., 2014. Circulation des modèles urbains, développement économique et géopolitique : la stratégie des relations internationales de Johannesburg. Présentation au séminaire «Circulation des modèles urbains ", PRODIG, 10/02/2014.

Prévot M. 2015, Catholicisme social et urbanisme. Maurice Ducreux et la fabrique de la Cité (1924-1985). Rennes, PUR, préface de Gabriel Dupuy.

Raynaud-Desmet L., 2011. La fabrication de la ville durable entre conflit et participation : les activistes urbains écologistes en région parisienne. Mémoire de master 2 en géographie, Université ParisDiderot, dir. Douay N. et Rivière D.

Raynaud-Desmet L., 2012. La fabrication de la ville durable entre conflit et participation : les activistes urbains écologistes en région parisienne. L'information géographique, « Activismes urbains : engagement et militantisme ", Armand Colin, Paris, Vol. 76, n 1, p. 36-51.

Sassen S., 2001. Impacts of information technologies on urban economic and politics. International Journal of Urban and Regional Research, 25(2), p. 411 - 418. 
Sassen S., 2011. The Global Street: Making the Political. Globalizations 8, $\mathrm{n}^{\circ}$ 5, p. 565-571. www.possible-futures.org/2011/11/22/the-global-street-comes-to-wall-street/ \#sthash.ebddf3sC.dpuf

Sawhney N., de Klerk C., Malhotra S., 2015. Civic Engagement through DIY Urbanism and Collective Networked Action. Planning Practice \& Research, 30(3), p. 337-354.

Simay P., 2008. Une autre ville pour une autre vie. Henri Lefebvre et les situationnistes. Métropoles [En ligne], 4 | 2008, mis en ligne le 18 décembre 2008, consulté le 28 août 2015. http:// metropoles.revues.org/2902

Stone D., 2004. Transfer agents and global networks in the 'transnationalization' of policy. Journal of European Public Policy, 11 (3), June 2004, p. 545-566.

Vareilles S., Ginhoux B., Vincent C, 2015. Les usages informels et inattendus, quels positionnements et gestion par l'action publique? Collectif d'Action pour la Ville/Expérimentation et Réflexion In situ (COLAVERI) http://colaveri.com/les-usages-informels-et-inattendus-quels-positionnements-etgestion-par-laction-publique-sophie-vareilles-berangere-ginhoux-et-carine-vincent

Whyte W., 1980. The Social Life of Small Urban Spaces. Project For Public Spaces Inc.

\section{NOTES}

1. Cette revue interdisciplinaire de recherche urbaine avait lancé, en 2013 , un appel à articles intitulé : «Politiques alternatives de développement urbain»: http://metropoles.revues.org/ 4622.

2. C'est le premier photographe à avoir utilisé la technique du time lapse pour filmer les mouvements, les mobilités et les interactions humaines dans l'espace public. À partir de cette observation empirique il propose un catalogue de critères de design qui font, selon lui, d'un espace public aménagé, un espace vivant et social (Crombez, op. cit, p. 30). http:// www.brainpickings.org/2013/08/22/the-social-life-of-small-urban-spaces-whyte/

3. http://www.pps.org/about/

4. www.streetplans.org/leadership_mike_lydon.php

5. www.streetplans.org

6. www.facebook.com/lydonmic

7. http://twitter.com/mikelydon

8. www.cnu.org

9. http://uneven-growth.moma.org

10. Ces interventions prétendent intervenir ponctuellement sur des points névralgiques.

11. http://adamparsonsdesignthesis.blogspot.fr/2010/12/urban-acupuncture-marcocasagrande.html ; https://www.facebook.com/pages/Casagrande-Laboratory/100874503486

12. https://www.facebook.com/Jaime-Lerner-106059656123844/

13. Cité par Crombez, 2014, p. 13.

14. http://www.bookclas.com/pdf/place-based-creative-problem-solving-and-the-power-ofthe.html

15. http://rebargroup.org

16. http://parkingday.org

17. Cet exemple a été pris par Dimitri Boutleux dans une conférence sur «l'urbanisme tactique » le 5 juin 2013 à Rennes Métropole. http://metropole.rennes.fr/les-conferences-de-rennesmetropole/viva-cites/l-urbanisme-tactique/ 
18. http://www.journaldunet.com/economie/transport/1172497-paris-tactical-urbanismplacemeter/ : «Placemeter ingests any kind of video to analyze pedestrian and vehicular movement, revealing hidden patterns and strategic opportunities » (https:// www.placemeter.com/about ).

19. http://www.journaldunet.com/economie/transport/1172497-paris-tactical-urbanismplacemeter/

20. Smart Cities. Efficace, innovante, participative : comment rendre la ville plus intelligente ?10 propositions pour faire émerger des villes intelligentes à la française, Institut de l'entreprise, 2013, cf. http://www.institut-entreprise.fr/les-publications/smart-cities-efficace-innovanteparticipative-comment-rendre-la-ville-plus

21. http://www.jardinons-ensemble.org/spip.php?article211

22. http://www.sfgate.com/realestate/article/Efforts-to-turn-empty-lots-to-a-glass-halffull-3219440.php

23. https://www.glasgow.gov.uk/index.aspx?articleid=17974

24. http://acteursduparisdurable.fr/actus/du-vert-pres-de-chez-moi

25. http://www.paris.fr/accueil/accueil-paris-fr/dans-ma-rue-l-appli-qui-ameliore-la- ville/ rub_1_actu_132205_port_24329; $\quad$ https://teleservices.paris.fr/sira/jsp/site/Portal.jsp? page $=$ formengine $\&$ form $=$ signalement

26. http://www.theguardian.com/commentisfree/2014/jan/19/urban-vibrancy-social-cleansinggentrification

27. http://www.paris.fr/permisdevegetaliser

28. http://www.huffingtonpost.fr/2016/05/08/nuit-debout-paris-construction-cinema-placerepublique-paris_n_9865302.html

29. C'est le cas des fundraising aux États-Unis dont une des réalisations les plus emblématiques a été le High Line Park de New York et son entretien grâce aux fonds collectés auprès des membres de l'association « Friends of the High Line » (Orduna-Giro P., Jacquot S., 2014)

30. http://www.cityrepair.org/intersection-repair-examples/

31. Voir la mission de réflexion sur "l'aménagement du territoire en France : refonder les relations entre l'État et les collectivités territoriales » confiée à Claudy Lebreton par le Premier ministre : https://www.parlement-et-citoyens.fr/project/comment-refonder-la-democratielocale/presentation/presentation-1 ou une expérience locale dans le Nord de la France : «A Loos en Gohelle, la démocratie impliquante », La Voix du Nord, 1/3/2016.

\section{ABSTRACTS}

Tactical planning provides every citizen to act materially on its everyday and immediate urban environment and to make it more pleasant, without waiting for the authorities / stakeholders responsible for planning and urbanism to answer his aspirations. At first glance it calls into question the central role of public institutions in the regulation and control of the public space and amenities. In this sense, the societal conditions of the emergence of these new initiatives of citizen ownership of public spaces, abandoned or not, must be analyzed. If the tactical planning endeavors to show that everyone has the capacity to transform, at the local level, how to think and plan the city, not as in his own name but on behalf of interest shared by other members of his community, alternative urban model relies thus on a large community connected to social 
networks that allow the movement to adopt new practices and initiatives and to adapt it in different local contexts. The tactical urbanism carries the standard of disinterested exchanges and values promoted by the pioneers of the digital vector of an active democracy or it is only the new faces of a mainstream solutions in urban planning? Finally we can question the transgressive and alternative characters because it seems to have been established as one of the aspects of conventional urban design solutions? The case of Paris illustrates the reception of the model and its gradual institutionalization.

L'urbanisme tactique propose à tout citoyen d'agir matériellement sur son environnement urbain immédiat et quotidien afin de le rendre plus agréable à vivre, et ce sans attendre que les autorités/acteurs en charge de l'aménagement et de l'urbanisme répondent à ses aspirations. À première vue cela remet en cause le rôle central des institutions publiques dans la régulation et la maîtrise de l'espace public et des aménagements. En ce sens, les conditions sociétales de l'émergence même de ces nouvelles initiatives d'appropriation citoyenne d'espaces publics, délaissés ou pas, doivent être analysées. Si l'urbanisme tactique s'attache à démontrer que chacun est en capacité de transformer, à l'échelle locale, la manière de penser et de faire la ville, ce n'est pas tant en son nom propre qu'au nom d'intérêts partagés par d'autres membres de sa communauté. Ce modèle urbain alternatif s'appuie donc sur une large communauté connectée aux réseaux sociaux qui permet la circulation des initiatives prises ou des pratiques adoptées et leurs adaptations dans différents contextes locaux. L'urbanisme tactique porte-t-il « l'étendard des échanges désintéressés et des valeurs du don promus par la génération des pionniers du Net » (Dagnaud) en étant le vecteur d'une démocratie « impliquante » ou ne serait-il désormais qu'un des visages renouvelés des solutions dominantes (mainstream) - d'aménagement urbain? Le cas de Paris permet d'illustrer cette réception du modèle et son institutionnalisation somme toute assez rapide.

\section{INDEX}

Mots-clés: activisme, urbanisme tactique, espace public

Keywords: actvism, tactical urbanism, public space

Subjects: Sur le Champ - Sur le Terrain

\section{AUTHORS}

\section{NICOLAS DOUAY}

Nicolas Douay, nicolas.douay@gmail.com, est Maître de conférences à l'Université Paris-Diderot et chercheur à Géographie-Cités UMR 8504.

\section{MARYVONNE PRÉVOT}

Maryvonne Prévot, prevot.maryvonne@neuf.fr, est Historienne, MCF-HDR en aménagement et urbanisme à Lille1 et membre du Laboratoire TVES (EA 4477). 